\title{
Effects of brief group mindfulness-based cognitive therapy for stress reduction among medical students in a Malaysian university
}

\begin{abstract}
It has been widely reported that medical students face considerable stress in medical school. In Malaysia, a brief (four-session, $2 \mathrm{~h}$ per week) group Mindfulness-based Cognitive Therapy (b-GMBCT/Mindful-Gym) was developed to help medical students cope with stress. The aim of this study was to evaluate the feasibility and effectiveness of the program in reducing stress among medical students in a Malaysian university. This was a single-group, prospective study. A total of 135 year-four medical students in psychiatric postings participated in the program (conducted in seven batches over 2 years). The following outcome variables were measured pre- and post-intervention: mindfulness, perceived stress, and general psychological distress. Intention-to-treat analyses showed significant reductions in perceived stress $(\mathrm{M}=-3.85, \mathrm{SD}=5.70,95 \% \mathrm{CI},-2.88$ to $-4.82, \mathrm{p}<0.001)$ and increase in mindfulness $(\mathrm{M}=0.46, \mathrm{SD}=0.80,95 \% \mathrm{CI}, 0.32$ to $0.59, \mathrm{p}<0.001)$ with medium effect sizes from pre- to post-intervention. The percentage of participants who reported having significant general psychological distress $(\mathrm{GHQ} \geq 4)$ reduced $(\mathrm{p}<0.001)$ from $36 \%(\mathrm{n}=48)$ at preintervention to $10 \%(\mathrm{n}=14)$ after the program. Although there were significant reductions in perceived stress among Malay and non-Malay medical students, Malay students had significantly lower level of perceived stress $(p=0.03)$ after the program. This study found that the $b-G M B C T$ is potentially an effective stress reduction program for medical students in Malaysia.
\end{abstract}

Keyword: Cognitive therapy; Medical students; Mental health; Mindfulness; Psychological distress; Stress 